\title{
MEAN GROWTH AND SMOOTHNESS OF ANALYTIC FUNCTIONS
}

\section{A. MATHESON}

ABstract. Let $G_{\alpha}$ denote the class of functions $f(z)$ analytic in the unit disk such that

$$
\int_{0}^{1}(1-r)^{-\alpha} M_{\infty}\left(f^{\prime}, r\right) d r<\infty,
$$

for some $\alpha(0<\alpha<1)$. A characterization of $G_{\alpha}$ is given in terms of moduli of continuity and an application is given to Riesz factorization of functions in $G_{\alpha}$.

1. Let $G_{\alpha}, 0<\alpha<1$, denote the class of functions $f$ analytic in the open unit disk $U$ which satisfy

$$
\psi_{\alpha}(f)=\int_{0}^{1}(1-r)^{-\alpha} M_{\infty}\left(f^{\prime}, r\right) d r<\infty,
$$

where $M_{\infty}\left(f^{\prime}, r\right)=\max \left\{\left|f^{\prime}\left(r e^{i \theta}\right)\right| ; 0 \leq \theta \leq 2 \pi\right\}$. Defining $\|f\|=\|f\|_{\infty}+\psi_{\alpha}(f)$ yields a norm on $G_{\alpha}$ which is easily seen to make $G_{\alpha}$ into a Banach algebra. The purpose of this paper is to give a characterization of the functions of $G_{\alpha}$ in terms of the smoothness of their boundary functions. In the first place it is easily seen that the functions of $G_{\alpha}$ satisfy a Lipschitz condition of order $\alpha$. In fact, somewhat more is true, as the following lemma shows. If $f$ is analytic in $U$ and continuous on $\bar{U}$, let $\omega(f, \delta)$ denote the modulus of continuity of $f$ on the boundary of $U$.

LEMMA 1. If $f \in G_{\alpha}$, then $f$ has a continuous extension to $\bar{U}$, and $\omega(f, \delta)=$ $o\left(\delta^{\alpha}\right)$.

ProOF. By a well-known theorem of Hardy and Littlewood [3, p. 263], it suffices to show that $M_{\infty}\left(f^{\prime}, r\right)=o\left((1-r)^{\alpha-1}\right)$. But $M_{\infty}\left(f^{\prime}, r\right)$ is nondecreasing, so this follows from the integrability condition.

The following theorem gives a precise condition on the modulus of continuity of a function for membership in the class $G_{\alpha}$. The theorem will be proved through a series of lemmas in $\S 2$ and an application will be given in $\S 3$.

THEOREM 1. Let $f$ be analytic in $U$ and continuous on $\bar{U}$. Then the following conditions are equivalent.

(1) $\psi_{\alpha}(f)<\infty$,

(2) $\int_{0}^{1} t^{-1-\alpha} \omega(f, t) d t<\infty$,

(3) $\sum_{n=1}^{\infty} 2^{n \alpha} \omega\left(f, 2^{-n}\right)<\infty$.

2. The first two lemmas of this section relate the modulus of continuity of a function $f$ with the growth of its derivative. It would be surprising if these results

Received by the editors August 8, 1980 and, in revised form, March 18, 1981.

1980 Mathematics Subject Classification. Primary 46J15. 
were not known, but the proofs, which follow closely the proof of the theorem of Hardy and Littlewood cited above, will be included for completeness. First let $\omega(\delta)$ be an increasing continuous function for $0 \leq \delta<\infty$, with $\omega(0)=0$ and $\omega(\delta)=$ $\omega(\pi)$ when $\delta \geq \pi$. Define

$$
\tilde{\omega}(\delta)=\delta \int_{\delta}^{\infty} t^{-2} \omega(t) d t \quad \text { and } \quad \hat{\omega}(\delta)=\int_{0}^{\delta} t^{-1} \omega(t) d t .
$$

Note that $\hat{\omega}(\delta) \equiv \infty$ may happen, but it is easy to see that $\omega(\delta) \leq \tilde{\omega}(\delta)$ for all $\delta$, and if $\omega(\delta)=o\left(\delta^{\beta}\right)$ for some $\beta$ with $0<\beta<1$, then $\tilde{\omega}(\delta)=o\left(\delta^{\bar{\beta}}\right)$ and $\hat{\omega}(\delta)=$ $o\left(\delta^{\beta}\right)$. In particular, if $f \in G_{\alpha}$, then $\hat{\omega}(f, \delta)$ is finite.

LEMMA 2. Let $f$ be analytic in $U$ and continuous on $\bar{U}$, and let $\omega(\delta)$ denote the modulus of continuity of $f$ on the boundary of $U$. Then

$$
M_{\infty}\left(f^{\prime}, r\right)=O(\tilde{\omega}(1-r) /(1-r)) \quad \text { as } r \rightarrow 1^{-} \text {. }
$$

Proof. Fix a point $z=r e^{i t}$ in the unit disk $U$. Then

$$
\begin{aligned}
f^{\prime}(z) & =\frac{1}{2 \pi} \int_{-\pi}^{\pi} f\left(e^{i \theta}\right) \frac{e^{i \theta}}{\left(e^{i \theta}-z\right)^{2}} d \theta \\
& =\frac{1}{2 \pi} \int_{-\pi}^{\pi}\left[f\left(e^{i \theta}\right)-f\left(e^{i t}\right)\right] \frac{e^{i \theta}}{\left(e^{i \theta}-z\right)^{2}} d \theta
\end{aligned}
$$

so that

$$
\left|f^{\prime}(z)\right| \leq \frac{1}{\pi} \int_{0}^{\pi} \frac{\omega(\delta)}{1-2 r \cos \delta+r^{2}} d \delta
$$

Now if $\frac{1}{2} \leq r<1$, and if $|\delta| \leq \pi$, then $1-2 r \cos \delta+r^{2} \geq(1-r)^{2}+2 \delta^{2} / \pi^{2}$. Thus

$$
\begin{aligned}
\left|f^{\prime}(z)\right| & \leq \frac{1}{\pi} \int_{0}^{\pi} \frac{\omega(\delta)}{(1-r)^{2}+2 \delta^{2} / \pi^{2}} d \delta \\
& \leq \frac{1}{\pi} \int_{0}^{1-r} \frac{\omega(\delta)}{(1-r)^{2}} d \delta+\frac{\pi}{2} \int_{1-r}^{\pi} \frac{\omega(\delta)}{\delta^{2}} d \delta \\
& \leq \frac{1}{\pi} \cdot \frac{\omega(1-r)}{1-r}+\frac{\pi}{2} \frac{\tilde{\omega}(1-r)}{1-r} \leq\left(\frac{1}{\pi}+\frac{\pi}{2}\right) \frac{\tilde{\omega}(1-r)}{1-r}
\end{aligned}
$$

since $\omega(1-r) \leq \tilde{\omega}(1-r)$. This completes the proof.

LEMMA 3. Let $f(z)$ be a function analytic in the unit disk $U$ such that

$$
f^{\prime}(z)=O(\phi(1-|z|) /(1-|z|)) \text { as }|z| \rightarrow 1^{-},
$$

where $\phi(\delta)$ is a nondecreasing function such that $\phi(\delta) \rightarrow 0$ and $\hat{\phi}(\delta) \rightarrow 0$ as $\delta \rightarrow 0$. Then $f$ can be extended to a continuous function on the closed unit disk $\bar{U}$ such that $\omega(f, \delta)=O(\phi(\delta)+\hat{\phi}(\delta))$.

Proof. Since $\hat{\phi}(\delta) \rightarrow 0$ as $\delta \rightarrow 0, \delta^{-1} \phi(\delta)$ is integrable on $0 \leq \delta \leq 1$, and so $f^{\prime}(z)$ is integrable over any radius of the unit disk. In particular $f(z)$ has finite radial limits everywhere on the unit circle.

Now let $\theta$ and $\theta^{\prime}$ be two real numbers such that $0<\theta^{\prime}-\theta<1$ and choose $r$ so that $1-r=\theta^{\prime}-\theta$. Let $C_{1}$ be the radial segment from $e^{i \theta}$ to $r e^{i \theta}, C_{2}$ the circular arc from $r e^{i \theta}$ to $r e^{i \theta^{\prime}}$, and $C_{3}$ the radial segment from $r e^{i \theta^{\prime}}$ to $e^{i \theta^{\prime}}$. If $C$ is 
the contour consisting of $C_{1}$ followed by $C_{2}$ followed by $C_{3}$, then $f\left(e^{i \theta^{\prime}}\right)-f\left(e^{i \theta}\right)=$ $\int_{C} f^{\prime}(z) d z$. Thus to estimate $\left|f\left(e^{i \theta^{\prime}}\right)-f\left(e^{i \theta}\right)\right|$ it suffices to estimate the integral of $f^{\prime}(z)$ separately over the contours $C_{1}, C_{2}$ and $C_{3}$. On $C_{1}$,

$$
\left|\int_{C_{1}} f^{\prime}(z) d z\right| \leq \int_{C_{1}}\left|f^{\prime}(z)\right||d z| \leq C \int_{r}^{1} \frac{\phi(1-s)}{1-s} d s=C \hat{\phi}(1-r) .
$$

Similarly, $\left|\int_{C_{3}} f^{\prime}(z) d z\right| \leq C \hat{\phi}(1-r)$. On $C_{2}$,

$$
\left|\int_{C_{2}} f^{\prime}(z) d z\right| \leq C \frac{\phi(1-r)}{1-r}\left|\theta-\theta^{\prime}\right|=C \phi(1-r) .
$$

Combining these estimates completes the proof.

LEMMA 4. Let $f$ be continuous on $\bar{U}$ and analytic in $U$. Then

$$
\int_{0}^{1}(1-r)^{-\alpha} M_{\infty}\left(f^{\prime}, r\right) d r \leq C_{1} \int_{0}^{1} t^{-1-\alpha} \omega(f, t) d t+C_{2} \omega(f, \pi),
$$

for some constants $C_{1}$ and $C_{2}$ depending only on $\alpha$.

Proof. Let $\omega(t)=\omega(f, t)$. By Lemma 2, $M_{\infty}\left(f^{\prime}, r\right) \leq C_{0} \tilde{\omega}(1-r) /(1-r)$ for some absolute $C_{0}$. Thus

$$
\begin{aligned}
\int_{0}^{1}(1-r)^{-\alpha} M_{\infty}\left(f^{\prime}, r\right) d r & \leq C_{0} \int_{0}^{1}(1-r)^{-\alpha-1} \tilde{\omega}(1-r) d r \\
& =C_{0} \int_{0}^{1} t^{-\alpha-1} \tilde{\omega}(t) d t \\
& =C_{0} \int_{0}^{1} t^{-\alpha} \int_{t}^{\infty} s^{-2} \omega(s) d s \\
& =C_{0} \int_{0}^{\infty} \int_{0}^{s \wedge 1} t^{-\alpha} d t s^{-2} \omega(s) d s \\
& =\frac{C_{0}}{1-\alpha} \int_{0}^{\infty}(s \wedge 1)^{1-\alpha} s^{-2} \omega(s) d s \\
& =\frac{C_{0}}{1-\alpha}\left[\int_{0}^{1} s^{-\alpha-1} \omega(s) d s+\int_{1}^{\infty} s^{-2} \omega(s) d s\right] \\
& =\frac{C_{0}}{1-\alpha}\left[\int_{0}^{1} s^{-\alpha-1} \omega(s) d s+\omega(\pi)\right] .
\end{aligned}
$$

Here $s \wedge 1=\min (s, 1)$. This proves the lemma.

LEMMA 5. Let $\omega$ be a nondecreasing function for $0 \leq t \leq 1$. Then there exist positive constants $c$ and $C$ depending only on $\alpha$ such that

$$
c \sum_{n=0}^{\infty} 2^{n \alpha} \omega\left(2^{-n}\right) \leq \int_{0}^{1} t^{-1-\alpha} \omega(t) d t \leq C \sum_{n=0}^{\infty} 2^{n \alpha} \omega\left(2^{-n}\right) .
$$

ProOF. Evidently

$$
\frac{1-2^{-\alpha}}{\alpha} 2^{n \alpha} \omega\left(2^{-n}\right) \leq \int_{2^{-n}}^{2^{-n+1}} t^{-1-\alpha} \omega(t) d t \leq \frac{2^{\alpha}-1}{\alpha} 2^{(n-1) \alpha} \omega\left(2^{-n+1}\right),
$$

and the lemma follows on summation. 
LEMMA 6. If $f \in G_{\alpha}$ then $\int_{0}^{1} t^{-1-\alpha} \omega(f, t) d t<\infty$.

Proof. For $n=1,2, \ldots$, choose $t_{n}$ and $t_{n}^{\prime}$ so that $\left|t_{n}-t_{n}^{\prime}\right|=2^{-n}$ and $\omega\left(2^{-n}\right)=\left|f\left(e^{i t_{n}}\right)-f\left(e^{i t_{n}^{\prime}}\right)\right|$. Let $r_{n}=1-2^{-n}$ and let $C_{n}$ be the contour from $e^{i t_{n}}$ to $e^{i t_{n}^{\prime}}$ constructed as in the proof of Lemma 3. Since

$$
f\left(e^{i t_{n}^{\prime}}\right)-f\left(e^{i t_{n}}\right)=\int_{C_{n}} f^{\prime}(z) d z,
$$

it follows that

$$
\begin{aligned}
\omega\left(2^{-n}\right)= & \left|f\left(e^{i t_{n}^{\prime}}\right)-f\left(e^{i t_{n}}\right)\right| \leq \int_{C_{n}}\left|f^{\prime}(z)\right||d z| \\
& \leq 2 \int_{r_{n}}^{1} M_{\infty}\left(f^{\prime}, r\right) d r+2^{-n} M_{\infty}\left(f^{\prime}, r_{n}\right) .
\end{aligned}
$$

Thus

$$
\sum_{n=0}^{\infty} 2^{n \alpha} \omega\left(2^{-n}\right) \leq 2 \sum_{n=0}^{\infty} 2^{n \alpha} \int_{r_{n}}^{1} M_{\infty}\left(f^{\prime}, r\right) d r+\sum_{n=0}^{\infty} 2^{n(\alpha-1)} M_{\infty}\left(f^{\prime}, r_{n}\right) .
$$

But

$$
\begin{aligned}
\sum_{n=0}^{\infty} 2^{n \alpha} \int_{r_{n}}^{1} M_{\infty}\left(f^{\prime}, r\right) d r & =\sum_{n=0}^{\infty} 2^{n \alpha} \sum_{k=n}^{\infty} \int_{r_{k}}^{r_{k+1}} M_{\infty}\left(f^{\prime}, r\right) d r \\
& =\sum_{k=0}^{\infty} \sum_{n=0}^{k} 2^{n \alpha} \int_{r_{k}}^{r_{k+1}} M_{\infty}\left(f^{\prime}, r\right) d r \\
& \leq \frac{2^{\alpha}}{2^{\alpha}-1} \sum_{k=0}^{\infty} 2^{k \alpha} \int_{r_{k}}^{r_{k+1}} M_{\infty}\left(f^{\prime}, r\right) d r \\
& \leq \frac{2^{\alpha}}{2^{\alpha}-1} \sum_{k=0}^{\infty} \int_{r_{k}}^{r_{k+1}}(1-r)^{-\alpha} M_{\infty}\left(f^{\prime}, r\right) d r \\
& =\frac{2^{\alpha}}{2^{\alpha}-1} \int_{0}^{1}(1-r)^{-\alpha} M_{\infty}\left(f^{\prime}, r\right) d r
\end{aligned}
$$

and

$$
\begin{aligned}
\sum_{n=0}^{\infty} 2^{n(\alpha-1)} M_{\infty}\left(f^{\prime}, r_{n}\right) & \leq \sum_{n=0}^{\infty} \int_{r_{n}}^{r_{n+1}}(1-r)^{-\alpha} M_{\infty}\left(f^{\prime}, r\right) d r \\
& =\int_{0}^{1}(1-r)^{-\alpha} M_{\infty}\left(f^{\prime}, r\right) d r .
\end{aligned}
$$

Thus the lemma follows from Lemma 5.

The proof of Theorem 1 is now immediate. The equivalence of (2) and (3) follows from Lemma 5. Lemma 6 is the implication (1) implies (2), and Lemma 4 guarantees that (2) implies (1).

3. As an application of the above analysis, this section describes certain factorization properties of the spaces $G_{\alpha}$. The next theorem is the basic result. 
THEOREM 2. Let $s \in H^{\infty}$ and $f \in G_{\alpha}$, and define

$$
T_{s} f(z)=\frac{1}{2 \pi i} \int_{|w|=1} \frac{f(w) \overline{s(w)}}{w-z} d w .
$$

Then $T_{s} f \in G_{\alpha}$, and there is a constant $C$ depending only on $\alpha$ such that $\left\|T_{s} f\right\| \leq$ $C\|s\|_{\infty}\|f\|$.

Proof. For $z \in U$, let $\hat{z}=z|z|^{-1}$. Since

$$
\left(T_{s} f\right)^{\prime}(z)=\frac{1}{2 \pi i} \int_{|w|=1} \frac{f(w) \overline{s(w)}}{(w-z)^{2}} d w=\frac{1}{2 \pi i} \int_{|w|=1} \frac{[f(w)-f(\hat{z})] \overline{s(w)}}{(w-z)^{2}} d w
$$

it follows that

$$
\left|\left(T_{s} f\right)^{\prime}(z)\right| \leq \frac{\|s\|_{\infty}}{2 \pi} \int_{|w|=1} \frac{|f(w)-f(\hat{z})|}{|w-z|^{2}}|d w| .
$$

Thus, using the argument of Lemma 2,

$$
M_{\infty}\left(\left(T_{s} f\right)^{\prime}, r\right) \leq C\|s\|_{\infty} \frac{\tilde{\omega}(f, 1-r)}{1-r},
$$

and the theorem follows as in the proof of Lemma 4.

Every function $f \in H^{\infty}$ can be factored as the product of an inner function and an outer function, and an inner function $G$ is said to divide $f$ if $f G^{-1} \in H^{\infty}$.

COROLLARY 1. If $f \in G_{\alpha}$ and $G$ is an inner function dividing $f$, then $f G^{-1} \in$ $G_{\alpha}$, and $\left\|f G^{-1}\right\| \leq C\|f\|$, where $C$ is the constant of Theorem 2 .

Proof. If $f G^{-1} \in H_{\infty}$, then $f G^{-1}$ has boundary values $f\left(e^{i \theta}\right) \overline{G\left(e^{i \theta}\right)}$, and $f G^{-1}=T_{G} f$.

COROLLARY 2. If $f \in G_{\alpha}$ and $f=G F$, where $G$ is inner and $F$ is outer, then $F \in G_{\alpha}$ and $\|F\| \leq C\|f\|$, where $C$ is the constant of Theorem 2.

Corollary 2 is analogous to a result of Samojan [2], who proves, essentially, that if $f$ has modulus of continuity $\omega(\delta)$, then $\omega(F, \delta)=O(\omega(\delta)+\tilde{\omega}(\delta))$, where $F$ is the outer factor of $f$. This should be compared with Theorem III, 13.30 of Zygmund [3, p. 121], and also the paper of Havin [1].

4. In conclusion several remarks are in order.

(1) The above analysis can be extended to integral moduli of continuity $\omega_{p}(f, \delta)$ $(1 \leq p<\infty)$ and to higher derivative. It should be noted that

$$
\int_{0}^{1}(1-r)^{-1} M_{\infty}\left(f^{\prime}, r\right) d r<\infty
$$

implies that $f$ is constant.

(2) If $u$ is continuous on $\bar{U}$ and harmonic in $U$, and satisfies $\int_{0}^{1} t^{-1-\alpha} \omega(u, t) d t<$ $\infty$, then the techniques above can be used to show that the modulus of continuity of its conjugate function $\tilde{u}$ satisfies the same condition. In particular the functions of $G_{\alpha}$ are characterized by their real parts.

(3) Since $G_{\alpha}$ is a Banach algebra of analytic functions, it is natural to ask for a description of its closed ideals. The description, which parallels the description for Lipschitz spaces of analytic functions, will be presented in a forthcoming paper. 
5. Part of the revision of this work was accomplished while the author was visiting the University of North Carolina at Chapel Hill.

\section{REFERENCES}

1. V. P. Havin, On the factorization of analytic functions smooth on the boundary, Zap. Nauxn. Sem. Leningrad. Otdel. Mat. Inst. Steklov. (LOMI) 22 (1971), 202-205.

2. F. A. Samojan, Division by an inner function in some spaces of functions analytic in the disk, Zap. Nauðn. Sem. Leningrad. Otdel. Mat. Inst. Steklov. (LOMI) 22 (1971), 206-208.

3. A. Zygmund, Trigonometric series, Cambridge Univ. Press, London and New York, 1968.

Department of Mathematics, OKLAhoma State UNIVERsity, Stillwater, OKLAHOMA 74078 